\title{
Towards Bathymetry-Optimized Doppler Re-navigation for AUVs
}

\author{
Ryan Eustice, Richard Camilli, and Hanumant Singh \\ Dept. of Applied Ocean Physics \& Engineering \\ Woods Hole Oceanographic Institution \\ Woods Hole, MA 02543 \\ Email: \{ryan,rcamilli,hanu\}@whoi.edu
}

\begin{abstract}
This paper describes a terrain-aided re-navigation algorithm for autonomous underwater vehicles (AUVs) built around optimizing bottom-lock Doppler velocity log (DVL) tracklines relative to a ship derived bathymetric map. The goal of this work is to improve the precision of AUV DVLbased navigation for near-seafloor science by removing the lowfrequency "drift" associated with a dead-reckoned (DR) Doppler navigation methodology. To do this, we use the discrepancy between vehicle-derived vs. ship-derived acoustic bathymetry as a corrective error measure in a standard nonlinear optimization framework. The advantage of this re-navigation methodology is that it exploits existing ship-derived bathymetric maps to improve vehicle navigation without requiring additional infrastructure. We demonstrate our technique for a recent AUV survey of largescale gas blowout features located along the U.S. Atlantic margin.
\end{abstract}

\section{INTRODUCTION}

Bottom-lock Doppler-based navigation is becoming a standard technique for underwater vehicle navigation [1], however, error grows as a function of percent distance traveled [2]. A traditional approach for removing this long-term "drift" has been to fuse bounded-error acoustic long-baseline (LBL) measurements via complementary filtering [3], [4]. Unfortunately, the time and effort of this solution may not be justified as LBL requires the deployment and calibration of transponder infrastructure [5]. Furthermore, it is becoming more and more routine to use AUVs in an exploratory context with missions of relatively short-duration (2-10 hours) spanning multiple, distant, survey sites [6]-[9]. For these type of missions, the setup, calibration, and eventual recovery of a LBL network represent a significant burden. Therefore, we seek alternative methods to remove Doppler drift.

This paper describes a terrain-optimized re-navigation algorithm for AUVs built around correcting bottom-lock DVL tracklines relative to a ship-derived bathymetric map. The goal of this work is to improve the precision of AUV DVLbased navigation for near-seafloor science by removing the low-frequency "drift" associated with a dead-reckon Doppler navigation methodology. Our strategy is to use ship-derived acoustic multibeam bathymetry as a corrective feedback source against which vehicle-derived bathymetry is compared - thus, "closing-the-loop" on DR drift. The advantage of this methodology is that it exploits existing bathymetric maps to improve vehicle navigation without requiring additional LBL infrastructure.

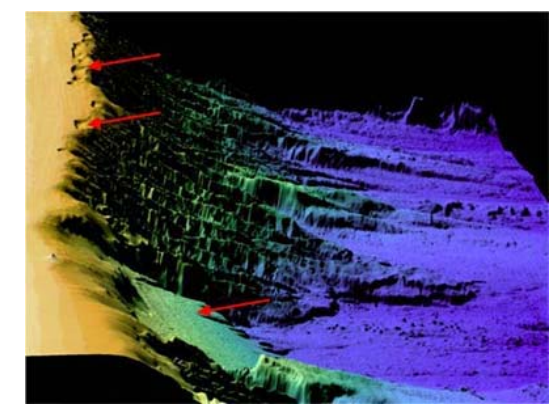

(a) Ship-derived multibeam bathymetry.

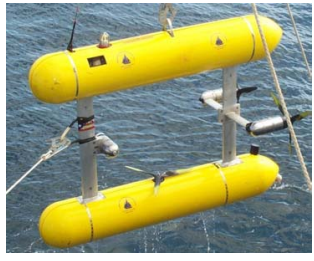

(b) The SeaBED AUV [11].
Fig. 1. A depiction of the science survey technology used. (a) An oblique view of a ship-derived acoustic multibeam map constructed from data taken along the continental shelf edge (the red arrows designate hypothesized methane cold-seep sites). This graphic emphasizes the fact that "slices" through the vertical topography should provide a distinct and useful mechanism for terrain-localization purposes. (b) A photo of the AUV employed for autonomous benthic surveying.

In the following, we begin our discussion by first describing the science-related application of this re-navigation technique to the study of continental-shelf methane cold-seeps [7], [10]. We then discuss the basic working principles of DVL-based navigation along with our simple corrective distortion model utilized for post-processing re-navigation. We show how this model can be used, along with vehicle-derived bathymetry (e.g. swath sensor, pencil-beam sonar, or even simply just vehicle altitude), to compute a discrepancy measure w.r.t. ship-derived multibeam data, and furthermore that this error can be minimized using a standard nonlinear least-squares optimization framework. Finally, we conclude with real-world results demonstrating the application of our technique to a recent AUV survey of large-scale gas blowout features located along the U.S. Atlantic margin.

\section{BACKGROUND}

\section{A. The Science Objective}

A shipboard program conducted in May 2000 provided major new insight into the origin of the enigmatic "crack"-like features arranged along a $40 \mathrm{~km}$ long stretch of the outermost shelf off Virginia and North Carolina [12]. High-resolution side-scan backscatter and chirp sub-bottom reflection data showed that the features were not simple normal faults, but 
appeared to be large-scale excavations or craters resulting from massive expulsion of gas through the seafloor.

In 2004, a multidisciplinary group of scientists from the Lamont-Doherty Earth Observatory, the Scripps Institute of Oceanography, and the Woods Hole Oceanographic Institution mounted an expedition to verify and characterize the gascharged fluid seepage, past and present, through the blowout craters, to determine the nature and origin of the gas [7], [10]. The fundamental question that was to be answered was whether there was present day discharge of gas-rich fluids through the floors or sidewalls of the blowouts, or were these suspected seepage sites relict features?

To gather data towards answering this question, the 2004 survey efforts focused on the following three different areas:

1) To acquire high-resolution shipboard multibeam bathymetry data over the blowouts and the surrounding area to provide the morphologic context for the seepage sites, and program the AUV mission (Fig. 1).

2) Survey suspected fluid discharge sites along the walls and floors of the blowout craters with the SeaBED AUV (Fig. 2), equipped with a high-resolution digital camera, a pencil-beam bathymetry mapper, and a dissolved methane sensor.

3) Collect precisely located gravity cores from the shelf edge delta for age control on the blowouts, to determine the nature of the gas (biogenic vs. thermogenic), and to measure pore water chemistry on samples from the suspected seepage sites.

\section{B. The Navigation Discrepancy}

Early into the cruise, we noticed that the raw DVL-based AUV survey tracklines were not registering well with the georeferenced ship-derived multibeam map (Fig. 3(a)). Furthermore, since we were confident in the accuracy of the shipderived multibeam data, we suspected a problem in our AUV's DVL-based navigation.

When operating in bottom-track mode, the DVL should provide a measurement of vehicle velocity referenced w.r.t. the (assumed) static seafloor, which can then be integrated to obtain a DR vehicle position. Curiously, though, in our case the discrepancy between ship vs. vehicle-derived bathymetry suggested that the bottom-track velocity measurement was additionally picking up a component of water current velocity as well. This was evident by the fact that the world-referenced DR vehicle trajectories differed by several hundreds of meters from their expected trackline lengths. ${ }^{1}$ Moreover, this discrepancy was significantly more than the expected DR drift.

We found that by adding slight dc offsets to the measured Doppler velocities (e.g., dc offsets $\leq 6 \mathrm{~cm} / \mathrm{s}$ at desired course over ground (COG) vehicle speeds of $40 \mathrm{~cm} / \mathrm{s}$ ) that we could compensate for much of this effect (Fig. 3(b)). Furthermore, these dc offsets changed on a per-dive basis and seemed to be well correlated with the ship-based acoustic Doppler current

\footnotetext{
${ }^{1}$ Ship-board ultra-short-baseline (USBL) vehicle tracking confirmed the existence of this discrepancy.
}

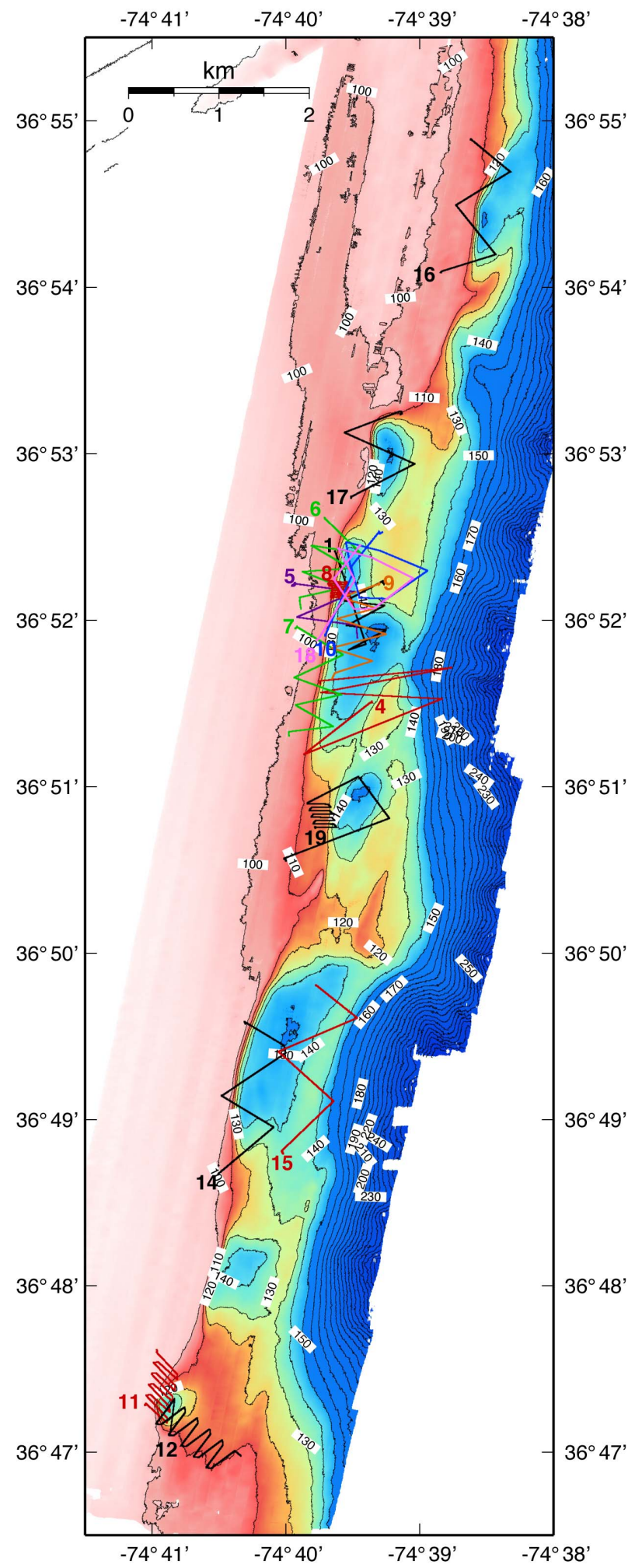

Fig. 2. An illustration of the survey area and associated AUV transects. Shown in the background is the ship-derived bathymetry, which was constructed using SM2000 multibeam sonar data. Overlaid in the foreground are the (numbered) AUV tracklines corresponding to 16 unique deployments (successful dives correspond to numberings $\{1,4-12,14-19\})$. 

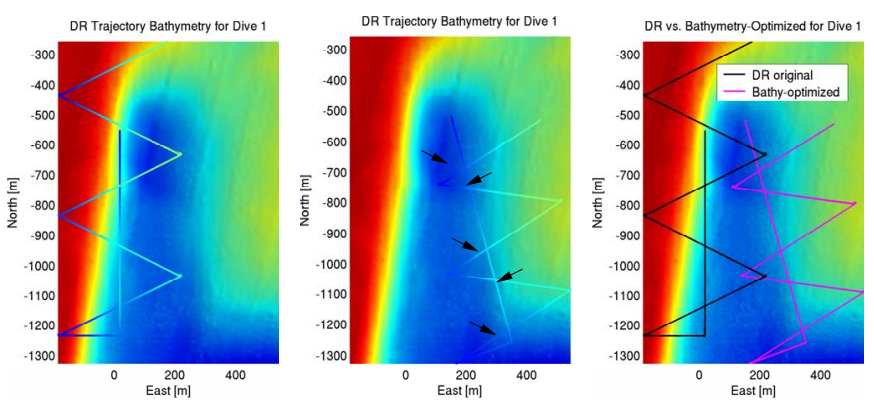

(a) DR trackline.

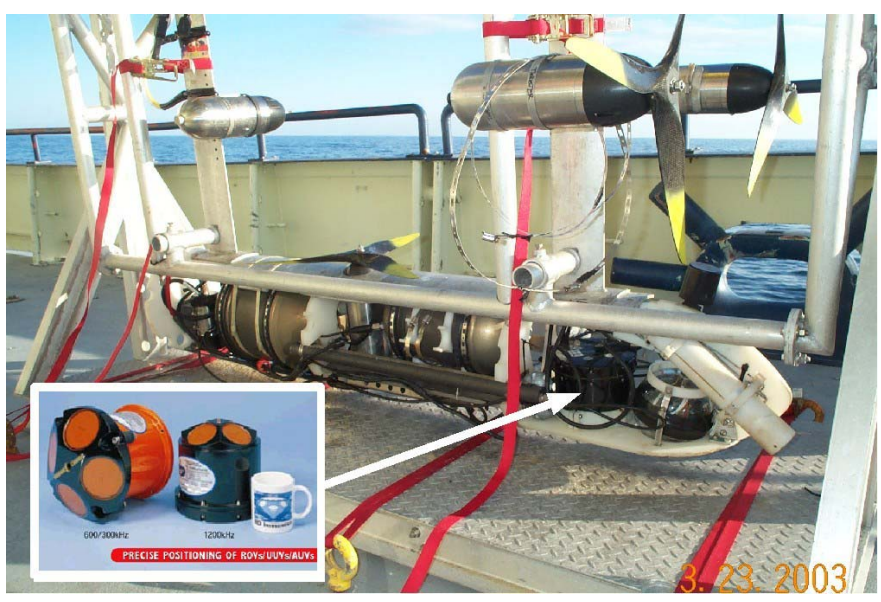

Fig. 4. A RD Instruments $1200 \mathrm{kHz}$ Workhorse Navigator DVL is shown in-situ on the bottom hull of the SeaBED AUV (outer hydrodynamic shell removed). figures plots the vehicle-derived bathymetry along the original DVL-obtained path, where shown in the background in the ship-derived multibeam map. Note that the vehicle-derived trackline bathymetry does not agree well with the shipderived multibeam data. (b) This figure plots the vehicle-derived bathymetry for the terrain-optimized trajectory. Notice how the vehicle bathymetry now agrees with the ship multibeam data, and furthermore, is self-consistent at the indicated cross-over points (black arrows). (c) This graphic compares the original DVL generated trajectory (black) to that of the terrain-aided trajectory (magenta). Notice how the two tracklines are shifted by several hundred meters and also how the DR trackline is axially stretched relative to the optimized trackline.

profiler (ADCP) measurements of near-seafloor water column velocities. This led us to believe that the offsets were not just a static malfunction (i.e., configuration error or hardware error) of the DVL unit, but rather a real phenomenon. ${ }^{2}$ Our working hypothesis for the observed bottom-track velocity offset is that since the benthic interface was dominated by fine clay sediments, fine-scale particulates were easily re-suspended into the shear layer causing the bottom-track measurement to "lock-on" to this velocity layer. ${ }^{3}$

\section{BATHYMETRY-AIDED DOPPLER RE-NAVIGATION}

\section{A. Basic Principles of Bottom-Lock DVL Navigation}

The DVL provides a measurement of seafloor-referenced vehicle velocity, which can be integrated over time to provide XYZ positional information. The basic working principle behind these bottom-referenced velocity measurements is the acoustic Doppler effect, which states that a change in the observed sound pitch results from relative motion. This change in sound pitch is directly proportional to the relative radial velocity between the source and receiver and can be used to recover seafloor-referenced vehicle velocity. Additionally, a DVL can also be used to measure water-referenced velocities [13].

\footnotetext{
${ }^{2}$ We verified that the unit was indeed setup for bottom-track mode and not programmed for water-track by mistake.

${ }^{3}$ Note that the vehicle was flying in an terrain-following mode that maintained an altitude of approximately $3 \mathrm{~m}$ off the bottom. Hence, the measured water column component would of have to of been in the near-seafloor shear layer.
}

1) DVL Technology: Commercially available broadband DVLs (Fig. 4), as opposed to traditional continuous-tone DVLs, make use of time dilation to compute a velocity measurement from an ensemble of "discrete" pings. The use of time dilation results in a more accurate measurement of the Doppler shift with single ping velocity error standard deviations less than $1 \%$ [3]. When $n$-ping ensemble averaging is performed, the standard deviation further decreases as $1 / \sqrt{n}$ [13]. Most offthe-shelf DVLs use a Janus transducer configuration [2], which consists of four downward-looking acoustic transducers each oriented at $30^{\circ}$ from the vertical (see inset of Fig. 4). In this configuration, each transducer measures the sensor's velocity with respect to the seafloor as projected onto the centerline of its acoustic beam axis, resulting in four measurements of beam-component velocity:

$$
\mathbf{v}_{b}(t)=\left[v_{b_{1}}(t), v_{b_{2}}(t), v_{b_{3}}(t), v_{b_{4}}(t)\right]^{\top} .
$$

Here, each $v_{b_{i}}(t)$ represents a scalar measurement of the sensor velocity as projected along the $i^{\text {th }}$ beam axis (i.e., $v_{b_{i}}(t)=\hat{\mathbf{e}}_{b_{i}} \cdot \mathbf{v}_{s}(t)$ where $\hat{\mathbf{e}}_{i}$ is the unit vector in the $i^{t h}$ beam direction).

2) Dead-Reckoned DVL Navigation: The beam component velocity measurements can be mapped to a standard Cartesian fixed instrument frame by the static $4 \times 4$ instrument transformation matrix $M$ parameterized by the transducer geometry [14]:

$$
\mathbf{v}_{s}(t)=\left[\begin{array}{c}
v_{s_{x}}(t) \\
v_{s_{y}}(t) \\
v_{s_{z}}(t) \\
e(t)
\end{array}\right]=\operatorname{Mv}_{b}(t) .
$$

The XYZ components of $\mathbf{v}_{s}$ correspond to the Cartesian components of the bottom-referenced velocity vector as expressed in the instrument reference frame, while $e(t)$ is a normalized least-squares measure of velocity error. Discarding the error term $e(t)$, the resulting 3 -vector of instrument frame velocities, $\mathbf{v}_{s}^{\prime}(t)$, can be rotated into a locally-level coordinate frame 
aligned with the navigation frame:

$$
\mathbf{v}_{n}(t)={ }_{s}^{n} \operatorname{Rv}_{s}^{\prime}(t),
$$

via the $3 \times 3$ rotation matrix ${ }_{s}^{n} \mathrm{R}$, which is computed using measurements from onboard roll, pitch, and heading sensors. These navigation frame velocities can then be integrated to obtain a DR position estimate [3]:

$$
\mathbf{x}_{n}(t)=\mathbf{x}_{n}\left(t_{0}\right)+\int_{t_{0}}^{t} \mathbf{v}_{n}(\tau) d \tau
$$

\section{B. Trajectory Distortion Model}

Our approach for trying to compensate for the DR navigation discrepancy was to model a per-dive constant velocity bias in the East and North components and then apply this correction to the recorded trajectory. In addition, we also identified two other sources of error as contributing to the overall trackline bathymetry discrepancy: drop-site shift and flux-gate magnetic compass declination. In the following we discuss our simplified approach for modeling these trackline distortions, which accounts for most of the first-order observed navigation error while remaining computationally efficient to implement in a nonlinear cost optimization framework.

1) Velocity Offsets: To compensate for the effect of velocity offsets in the DR position estimate, we modeled the true COG vehicle velocity, $\mathbf{v}_{n}(t)$, as consisting of the measured Doppler vehicle velocity, $\tilde{\mathbf{v}}_{n}(t)$, (hypothesized to be referenced to the shear layer due to suspended particulates) plus a dc offset, $\mathbf{c}_{n}$, that accounts for the shear layer's own velocity over the bottom:

$$
\mathbf{v}_{n}(t)=\tilde{\mathbf{v}}_{n}(t)+\mathbf{c}_{n} .
$$

Inserting the above into (1) yields:

$$
\begin{aligned}
\mathbf{x}_{n}^{\prime}(t) & =\mathbf{x}_{n}\left(t_{0}\right)+\int_{t_{0}}^{t}\left(\tilde{\mathbf{v}}_{n}(\tau)+\mathbf{c}_{n}\right) d \tau \\
& =\mathbf{x}_{n}\left(t_{0}\right)+\int_{t_{0}}^{t} \tilde{\mathbf{v}}_{n}(\tau) d \tau+\left(t-t_{0}\right) \mathbf{c}_{n} \\
& =\tilde{\mathbf{x}}_{n}(t)+\left(t-t_{0}\right) \mathbf{c}_{n}
\end{aligned}
$$

where $\tilde{\mathbf{x}}_{n}(t)=\mathbf{x}_{n}\left(t_{0}\right)+\int_{t_{0}}^{t} \tilde{\mathbf{v}}_{n}(\tau) d \tau$ is the recorded DR trajectory and $\mathbf{x}_{n}^{\prime}(t)$ its corrected version.

2) Magnetic Declination: This error refers to the static offset between the measured compass reading and true North. Neglecting this effect results in a residual rotational alignment residing between the navigation frame and the true Northoriented world frame. Accounting for this alignment error in (2) we have:

$$
\begin{aligned}
\mathbf{x}_{w}^{\prime}(t) & ={ }_{n}^{w} \mathrm{R}\left(\theta_{d}\right) \mathbf{x}_{n}^{\prime}(t) \\
& ={ }_{n}^{w} \mathrm{R}\left(\theta_{d}\right)\left(\tilde{\mathbf{x}}_{n}(t)+\left(t-t_{0}\right) \mathbf{c}_{n}\right),
\end{aligned}
$$

where ${ }_{n}^{w} \mathrm{R}\left(\theta_{d}\right)$ is the rotation matrix corresponding to the magnetic declination, $\theta_{d}$. Additionally, because we were able to measure the shear layer water velocity using the ship-board ADCP prior to our AUV dives, we found that it more convenient to parameterize the dc velocity offset in the world frame as:

$$
\mathbf{c}_{n}={ }_{n}^{w} \mathrm{R}^{\top}\left(\theta_{d}\right) \mathbf{c}_{w},
$$

where $\mathbf{c}_{w}$ is the ship-based water current measurement. Incorporating this parametrization into (3) yields:

$$
\mathbf{x}_{w}^{\prime}(t)={ }_{n}^{w} \mathrm{R}\left(\theta_{d}\right) \tilde{\mathbf{x}}_{n}(t)+\left(t-t_{0}\right) \mathbf{c}_{w} .
$$

3) Drop-site Shift: The last modeled source of error in the DR estimate is that the vehicle launch-site origin may have been be off due to a lag between vehicle launch and vehicle descent. In other words, the vehicle drifted on the surface for several minutes before the mission actually started, thus causing the assumed survey origin in (2) (i.e., $\mathbf{x}_{n}\left(t_{0}\right)$ ) to be off by as much as a couple hundred meters. Accounting for this error requires that we simply shift the DR trackline estimate of (4) by an amount $\mathbf{x}_{O}$ :

$$
\begin{aligned}
\mathbf{x}_{w}^{\prime \prime}(t) & =\mathbf{x}_{w}^{\prime}(t)+\mathbf{x}_{o} \\
& ={ }_{n}^{w} \mathrm{R}\left(\theta_{d}\right) \tilde{\mathbf{x}}_{n}(t)+\left(t-t_{0}\right) \mathbf{c}_{w}+\mathbf{x}_{o} .
\end{aligned}
$$

4) Resulting 2D Distortion Model: Finally, because the AUV was able to measure bounded depth, $z_{w}(t)$, via an onboard Paroscientific depth sensor, we further restricted the DR trajectory correction of (5) to only consider the horizontal plane components:

$$
\begin{aligned}
& x_{w}^{\prime \prime}(t)=\tilde{x}_{n}(t) \cos \theta_{d}+\tilde{y}_{n}(t) \sin \theta_{d}+\left(t-t_{0}\right) c_{x}+x_{o} \\
& y_{w}^{\prime \prime}(t)=-\tilde{x}_{n}(t) \sin \theta_{d}+\tilde{y}_{n}(t) \cos \theta_{d}+\left(t-t_{0}\right) c_{y}+y_{o} .
\end{aligned}
$$

where

$\tilde{x}_{n}, \tilde{y}_{n}$ are the XY components of $\tilde{\mathbf{x}}_{n}(t)$,

$c_{x}, c_{y}$ are the XY components of $\mathbf{c}_{w}(t)$,

$x_{o}, y_{o}$ are the XY components of $\mathbf{x}_{o}$,

$\theta_{d} \quad$ is the compass declination.

\section{Terrain-Based Trackline Optimization}

In any DR methodology, because position estimation is performed in an open-loop manner, the error drift grows monotonically with time. When using a DVL, usual means for reseting this type of error are to fuse bounded-error LBL measurements at depth [3], [4] or, when working in shallow water, to make frequent surface maneuvers and obtain GPS fixes [15]. However, neither of these solutions is ideal for multiple, distant, short duration, AUV deployments as LBL requires deploying and recovering transponder infrastructure while GPS surfacings are impractical in deeper water. Since existing ship-derived bathymetric maps of the seafloor are often readily available, our methodology for bounding DR DVL drift (in post-processing) is to "close the navigation loop" by referencing the vehicle-derived trackline bathymetry to a georeferenced multibeam bathymetric map.

1) Bathymetry Assumptions: We assume that a standard ship-derived bathymetric map [16], [17], denoted $M_{w}$, exists:

$$
M_{w}[n, m]=Z\left(x_{w}[n], y_{w}[m]\right),
$$

where $Z(x, y)$ is the mean free-surface depth of the seafloor at location $(x, y), x_{w}[n], y_{w}[m]$ are the grid sample points, and $n, m$ are the sample integer indexes. In addition, we also assume that the AUV is able to make range and bearing measurements (e.g., multibeam sonar, pencil-beam sonar, or 
altitude sensor) from itself to the seafloor so that this information can be used to construct a swath of bathymetry directly beneath the vehicle trackline. For example, in our scenario of altitude-only measurements and a stable roll/pitch platform we can reconstruct a vehicle-derived bathymetric "slice", denoted $S_{w}$, by simply adding measured vehicle altitude to measured vehicle depth:

$$
\begin{aligned}
S_{w}\left[t_{i}\right] & =z_{w}\left[t_{i}\right]+A\left[t_{i}\right] \\
& \equiv Z\left(x_{w}^{\prime \prime}\left[t_{i}\right], y_{w}^{\prime \prime}\left[t_{i}\right]\right),
\end{aligned}
$$

where $z_{w}\left[t_{i}\right]$ is the measured vehicle depth at time index $t_{i}$, $A\left[t_{i}\right]$ is the measured vehicle altitude, $Z(x, y)$ is the mean freesurface depth of the seafloor at location $(x, y), x_{w}^{\prime \prime}\left[t_{i}\right], y_{w}^{\prime \prime}\left[t_{i}\right]$ are the distortion compensated trajectory samples computed from (6), and $\left\{t_{i}\right\} \in\left[t_{0}, t\right)$ is the set of recorded sample times.

2) Optimization Framework: In order to minimize the discrepancy between ship vs. vehicle-derived bathymetry for re-navigation purposes we utilized a standard nonlinear leastsquares Levenberg-Marquardt optimization framework [18, $\S 15.5]$. We defined our cost function as the summed squared difference between ship vs. vehicle derived bathymetry:

$$
C=\sum_{t_{i}}\left(\hat{S}_{w}\left[t_{i}\right]-S_{w}\left[t_{i}\right]\right)^{2},
$$

where

$$
\hat{S}_{w}\left[t_{i}\right]=\left.M_{w}\right|_{\left\{x_{w}^{\prime \prime}\left[t_{i}\right], y_{w}^{\prime \prime}\left[t_{i}\right]\right\}}
$$

is the gridded bathymetry map interpolated to the trajectory sample points (Algorithm 1), and optimization is performed over the parameter vector $\mathbf{p}=\left[c_{x}, c_{y}, x_{o}, y_{o}, \theta_{d}\right]$. Because we were able to obtain a good initial guess for both the magnetic declination (from a nautical chart) and the shear layer water velocity offsets (from the ship-board ADCP), but not necessarily the drop-site offsets, we decided to implement a hierarchal optimization approach whereby we first optimized over $x_{o}, y_{o}$ followed by full-scale optimization over $\mathbf{p}$.

Require: $M_{w}[n, m], x_{w}[n], y_{w}[m]$ \{gridded bathy. map

Require: $S_{w}\left[t_{i}\right]$ \{vehicle derived bathymetry\}

Require: $\tilde{x}_{n}\left[t_{i}\right], \tilde{y}_{n}\left[t_{i}\right]$ \{raw DR trajectory samples\}

Require: $x_{o}, y_{o}, c_{x}, c_{y}, \theta_{d}$ \{parameter values\}

1: compute $x_{w}^{\prime \prime}\left[t_{i}\right], y_{w}^{\prime \prime}\left[t_{i}\right]$ from $\tilde{x}_{n}\left[t_{i}\right], \tilde{y}_{n}\left[t_{i}\right]$ according to equation (6)

2: compute $\hat{S}_{w}\left[t_{i}\right]$ by interpolating $M_{w}[n, m]$ to the $x_{w}^{\prime \prime}\left[t_{i}\right], y_{w}^{\prime \prime}\left[t_{i}\right]$ sample points

3: compute the squared error cost $C$ according to (7)

4: return $C$

Algorithm 1: Bathymetry-based error measure.

\section{RESUlts}

In this section we show real-world results where we have applied our terrain-optimized re-navigation algorithm to dives $\{1,4-10\}$. We begin with Fig. 5(a), which plots the vehiclederived trackline bathymetry overlaid atop of the ship-derived
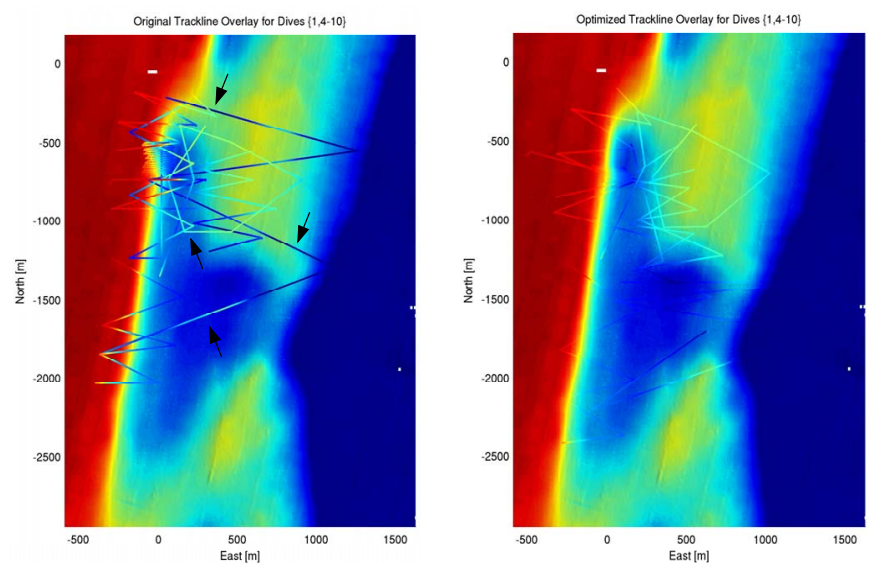

(a) Vehicle-derived vs. multibeam map bathymetry.
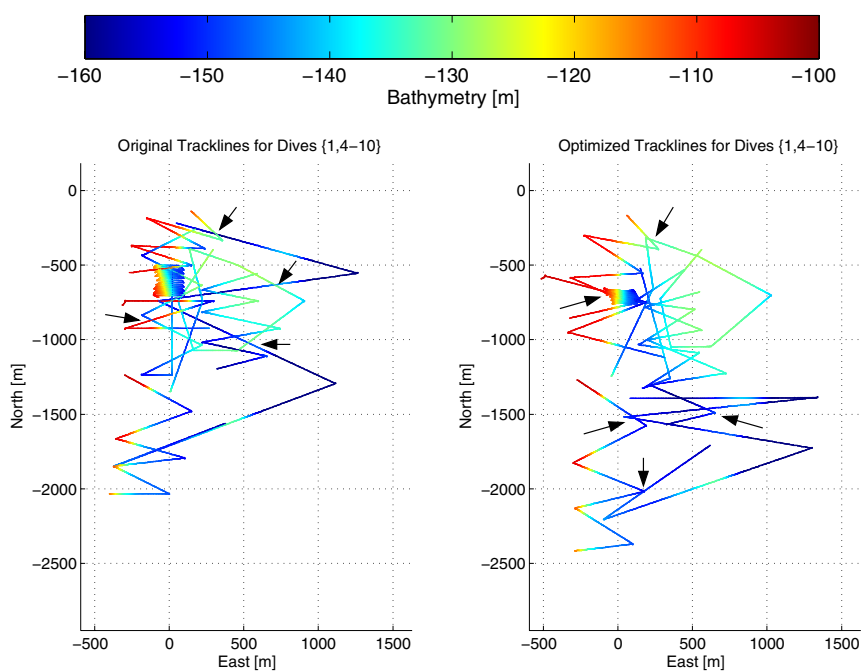

(b) Comparison of the cross-dive vehicle bathymetry.
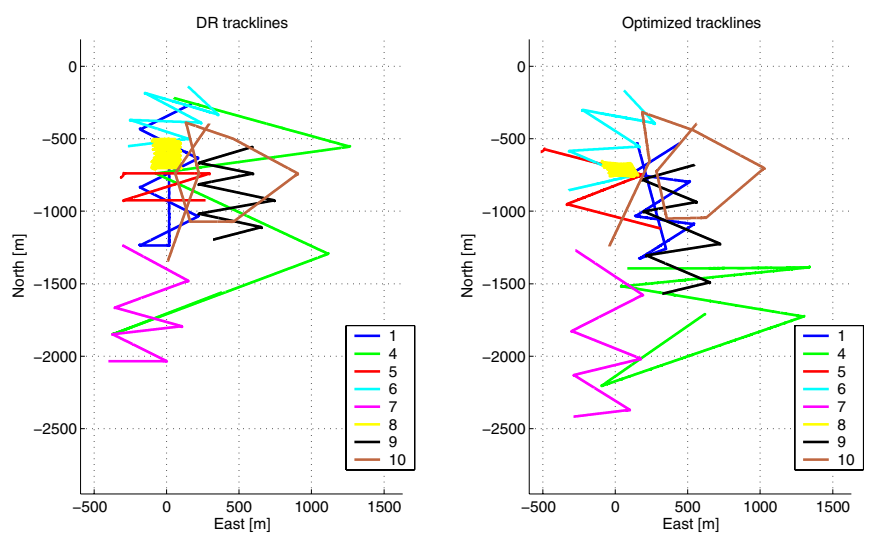

(c) DR vs. optimized tracklines.

Fig. 5. A comparison of the original vs. bathymetry-optimized vehicle tracklines for dives $\{1,4-10\}$. (a) AUV tracklines with vehicle-derived bathymetry overlaid on top of the ship-derived multibeam map. Note that there is a significant discrepancy between the multibeam map and the DR vehicle bathymetry (black arrows), however, the optimized trackline bathymetry agrees well. (b) These plots are the same as the previous, but without the multibeam map in the background for clarity. Notice how the optimized tracklines are in good bathymetric agreement across dives (black arrows). (c) A spatial comparison of the DR vs. optimized tracklines, color-coded by dive number for clarity. 
multibeam map. The leftmost plot shows tracklines corresponding to the raw DR trajectories as computed by the AUV. Notice how the vehicle-derived bathymetry in the basin is clearly in discrepancy with the multibeam data (indicated by black arrows). Furthermore, Fig. 5(b) shows that the overlapping portions of the cross-track/cross-dive trajectories are not in agreement (again indicated by black arrows). On the other hand, the terrain-optimized vehicle trajectories appear remarkably consistent. We note that the following three important results are worth noticing: 1) the terrain-optimized vehicle bathymetry closely agrees with the multibeam map, 2) both the cross-track and cross-dive overlapping portions of its trajectories are self-consistent, and 3) the magnitude of the error correction is substantial (Fig. 5(c)). Essentially, by pinning the DR trajectories to the low-frequency multibeam map we have accounted for most of the DR trackline error while still preserving the high-frequency DVL positional information. Fig. 6 further highlights this result by comparing the vehicle vs. interpolated multibeam bathymetry slices for each trackline.

\section{CONCLUSION}

In conclusion, this paper demonstrated a terrain-aided renavigation technique that is founded upon minimizing the discrepancy between vehicle-derived vs. ship-derived multibeam data of the seafloor. This can be used in post-processing to correct the recorded DR trajectory by constraining accumulated navigation error to the resolution of the bathymetric map via standard optimization techniques. We note that an advantage of using this methodology for localization is that it provides navigation constraints in a time-independent manner unlike other sensors, which may suffer from time-aliasing (e.g., water-packet dependent chemical sensors like methane concentration).

Finally, as a side-note we mention that this technique was developed while onboard our 2004 research cruise to counter the large DVL navigation errors that we were seeing during the cruise. Our objective then was to provide the science team with better geo-referenced navigation data for cruise-based analysis. Since then we have become aware of prior work in the literature regarding terrain-aided Bayesian inference techniques that appear to be statistically more principled [19], [20].

\section{ACKNOWLEDGMENTS}

This work was funded in part by the CenSSIS ERC of the National Science Foundation under grant EEC-9986821. This paper is WHOI contribution number 11400.

\section{REFERENCES}

[1] L. Whitcomb, "Underwater robotics: Out of the research laboratory and into the field," in Proc. IEEE Intl. Conf. Robot. Auto., 709-716, Apr. 2000.
[2] N. Brokloff, "Matrix algorithm for doppler sonar navigation," in Proc. OCEANS MTS/IEEE Conf. Exhib., vol. 3, Brest, France, Sept. 1994, pp. 378-383.

[3] L. Whitcomb, D. Yoerger, and H. Singh, "Advances in Doppler-based navigation of underwater robotic vehicles," in Proc. IEEE Intl. Conf. Robot. Auto., vol. 1, 1999, pp. 399-406.

[4] _ "Towards precision robotic maneuvering, survey and manipulation in unstructured undersea environments," in Proc. Intl. Symp. Robotics Research, Springer Verlag, London, 1998, pp. 45-54.

[5] M. Hunt, W. Marquet, D. Moller, K. Peal, W. Smith, and R. Spindel, "An acoustic navigation system," Woods Hole Oceanographic Institution, Tech. Rep. WHOI-74-6, Dec. 1974.

[6] H. Singh, R. Armstrong, F. Gilbes, R. Eustice, C. Roman, O. Pizarro, and J. Torres, "Imaging coral I: Imaging coral habitats with the SeaBED AUV," J. Subsurface Sensing Tech. Apps., vol. 5, no. 1, pp. 25-42, Jan. 2004.

[7] J. Hill, N. Driscoll, J. Weissel, M. Kastner, H. Singh, M. Cormier, R. Camilli, R. Eustice, R. Lipscomb, N. McPhee, K. Newman, G. Robertson, E. Solomon, and K. Tomanka, "A detailed near-bottom survey of large gas blowout structures along the US Atlantic shelf break using the autonomous underwater vehicle (AUV) SeaBED," in EOS, Trans. Am. Geophysical Union, Abstract, 2004, in Print.

[8] C. Langmuir, C. German, P. Michael, D. Yoerger, D. Fornari, T. Shank, P. Asimow, and H. Edmonds, "Hydrothermal prospecting and petrological sampling in the Lau Basin: Background data for the integrated study site," EOS, Trans. Am. Geophysical Union, Fall Meeting Abstracts, vol. 85, no. 47, pp. B13A-0189, 2004.

[9] B. Fletcher, "Chemical plume mapping with an autonomous underwater vehicle," in Proc. OCEANS MTS/IEEE Conf. Exhib., vol. 1, Honolulu, HI, USA, Nov. 2001, pp. 508-512.

[10] K. Newman, N. Driscoll, J. Weissel, M. Kastner, H. Singh, M. Cormier, R. Camilli, R. Eustice, R. Lipscomb, N. McPhee, J. Hill, G. Robertson, E. Solomon, and K. Tomanka, "A potential link between fluid expulsion and slope stability: Geochemical anomalies measured in the gas blowouts along the U.S. Atlantic margin provide new constraints on their formation," in EOS, Trans. Am. Geophysical Union, Abstract., 2004, in Print.

[11] H. Singh, R. Eustice, C. Roman, and O. Pizarro, "The SeaBED AUV - a platform for high resolution imaging," in Unmanned Underwater Vehicle Showcase, Southampton Oceanography Centre, UK, Sept. 2002.

[12] N. Driscoll, J. Weissel, and J. Goff, "Potential for large-scale submarine slope failure and tsunami generation along the U.S. mid-Atlantic coast," Geology, vol. 28, no. 5, pp. 407-410, 2000.

[13] RD Instruments, "Acoustic Doppler Current Profiler: Principles of operation a practical primer," RD Instruments, San Diego, CA, USA, Tech. Rep., 1996.

[14] — , "ADCP coordinate transformation," RD Instruments, San Diego, CA, USA, Tech. Rep., 1998.

[15] S. Smith and J. Park, "Navigational data fusion in the Ocean Explorer autonomous underwater vehicles," in Proc. Intl. Symp. Underwater Tech., Tokyo, Japan, Apr. 1998, pp. 233-238.

[16] C. de Moustier, "Beyond bathymetry: Mapping acoustic backscattering from the deep seafloor with Sea Beam," J. Acoustical Soc. Am., vol. 79, no. 2, pp. 316-331, Feb. 1986.

[17] B. Calder and L. Mayer, "Robust automatic multibeam bathymetric processing," in Proc. U.S. Hydrographic Conf., Norfolk, VA, 2001.

[18] W. Press, S. Teukolsky, W. Vetterling, and B. Flannery, Numerical Recipes in C: The Art of Scientific Computing, 2nd ed. Cambridge University Press, 1992.

[19] S. Williams, "A terrain-aided tracking algorithm for marine systems," in Proc. Intl. Conf. Field Service Robot., July 2003.

[20] N. Bergman, L. Ljung, and F. Gustafsson, "Terrain navigation using Bayesian statistics," IEEE Control Systems Magazine, vol. 19, no. 3, pp. 33-40, June 1999. 


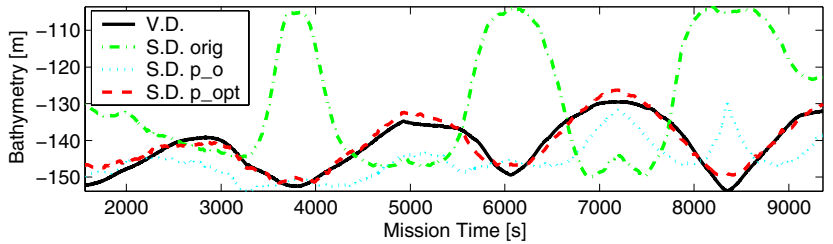

(a) Dive 01

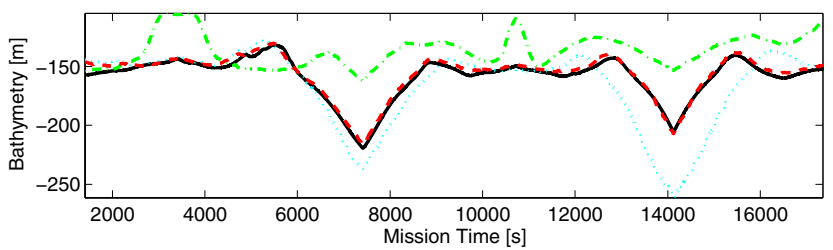

(b) Dive 04.

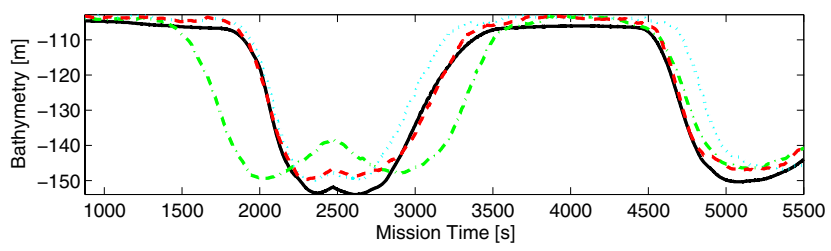

(c) Dive 05 .

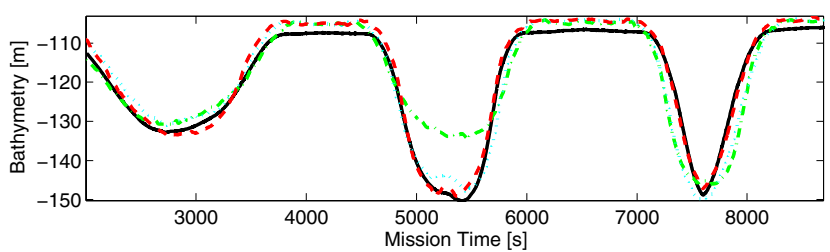

(d) Dive 06

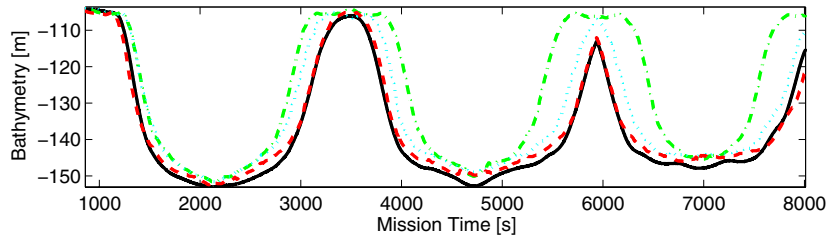

(e) Dive 07.

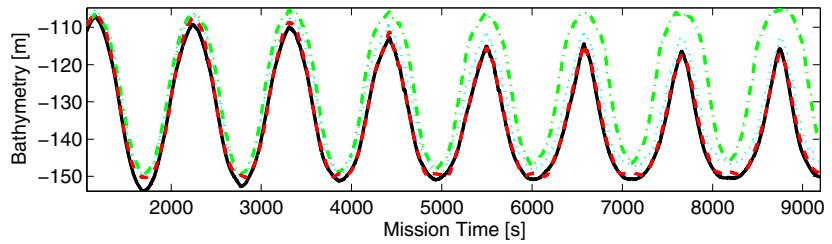

(f) Dive 08 .

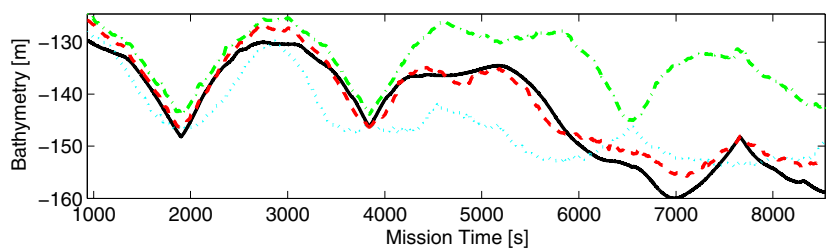

(g) Dive 09.

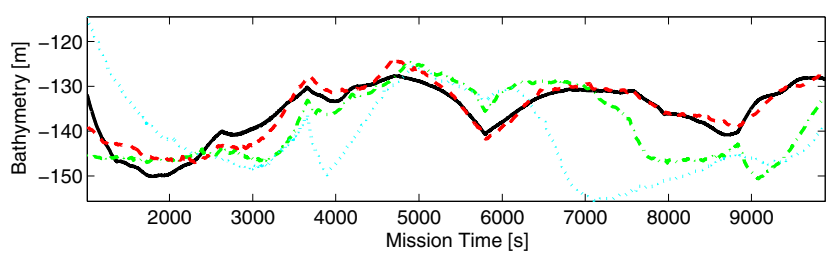

(h) Dive 10.

Fig. 6. A comparison of the vehicle-derived bathymetry "slices" vs. interpolated multibeam map values for the tracklines of Fig. 5. Plot (a) contains the legend where V.D. refers to the vehicle-derived bathymetry (black), S.D. orig refers to the ship-derived multibeam bathymetry interpolated to the raw DR trackline (green), S.D. $p_{\_} o$ is the ship-derived bathymetry interpolated to the trackline associated with our initial "best guess" of the distortion parameter vector p (cyan), and finally S.D. p_opt is the ship-derived bathymetry interpolated to the resulting optimized trackline (red). Notice the generally good agreement for all dives between the optimized trackline bathymetry to that measured by the vehicle. 